\title{
The Preliminary Study on the Mechanism about Piperine Regulating the Knee Osteoarthritis Based on Network Pharmacological Methods
}

\begin{abstract}
:
Objective: To explore the target of anti-knee osteoarthritis (KOA) in the effective chemical compounds of piper longum L based on network pharmacological methods.
\end{abstract}

Methods: The active chemical compounds of piper longum L were collected employing database retrieval on TCMSP, TCM-PTD, and literature mining. The Swiss Target Prediction service predicts the targets of active chemical compounds, and at the same time, the targets of the drugs treating knee osteoarthritis were collected by retrieving the OMIM and CTD databases. The targets were subjected to an alignment analysis to screen out piperine and we simulated the binding sites in vivo of compounds and proteins via AutoDock. After that, the rat models of knee osteoarthritis were established. The rats in model groups were given piperine treatment. The verification of the anti-KOA target PPARG and MAPK1 was done by Western blot and co-immunoprecipitation.

Results: Nine active ingredients were predicted. According to Lipinski's rule, piperine was speculated as a possible active ingredient. According to the possible targets of piperine and the KOA's possible targets, three co-targets of them were confirmed, PPARG and MAPK1 were related to knee osteoarthritis (KOA). Molecular docking results show that piperine can hinder the binding of PPARG protein ARG-212 and GLN-420 amino-acid residues to each other. After 20 weeks of piperine treating, Western blot found that piperine can significantly increase the 
expression level of PPARG and reduce the expression level of MAPK1 in model rats. The endogenous interaction between PPARG and MAPK1 was verified by co-immunoprecipitation.

Conclusion: Piper longum L can regulate the progression of knee osteoarthritis (KOA) by its active ingredient piperine, can affect the expression of PPARG and MAPK1 proteins, and PPARG and MAPK1 proteins have endogenous interactions.

Keywords: Piper longum L, Piperine, Network Pharmacology, Knee Osteoarthritis, PPARG

\section{INTRODUCTION}

Osteoarthritis (osteo arthritis, OA) are a progressive, degenerative joint disease that is common in the clinic, and the primary mechanism is that the cartilage tissue on the joint surface is damaged, causing joint pain and dysfunction. The current treatment can only be limited to symptomatic treatment ${ }^{1}$. Osteoarthritis attacks knee frequently, and the knee is a frequent part of osteoarthritis (OA). For knee osteoarthritis (KOA), Western medicine generally uses internal anti-inflammatory and analgesics and artificial joint replacement. Moreover, there are distinct disadvantages, such as temporary effect, numerous adverse reactions, many contraindications to artificial joint replacement, and high prices of it ${ }^{2-4}$. Mongolian medicine has accumulated rich experience in treating KOA. The doctors believe that the KOA belongs to the category of "yellow water disease of joints." Its pathogenesis is mainly that the predisposing factors including moisture, catching a chill, strenuous activity, over fatigue and other inducements cause dysfunction of Heyi, Sheila, Badakan, Yellow water, and others, the damage to the tissue around the joints or the accumulation of Xieri Wusu on joints causing the osteoarthritis." Red, swollen, pain" are the main symptoms. Mongolian medicine thinks of piper longum $\mathrm{L}$ as the 
medicine for Badakan, which can regulate the unbalance of three factors, activating blood circulation. It also works on exorcism righting, promoting blood circulation to remove blood stasis and immunoregulation ${ }^{5,6}$.

Mongolian medicine Naru Sanwei Pill is made from radix aconiti kusnezoffii praeparata, medicine terminalia fruit, and piper longum L. Piper longum $L$ is the essential medicine in this prescription due to its anti-inflammatory. Piper longum L often treats rheumatism, arthralgia and pain in waist and leg. Piperine is not only the significant component of piper longum $\mathrm{L}$ dissolved into blood, but also the active ingredient of piper longum L works on "reducing accretion," eliminating Xieri WuSu, dispelling wind, eliminating cold and pain in Mongolian medicine. Piperine has been proven by numerous studies to have antipyretic, anti-inflammatory, antioxidant, antiendotoxin, immunoregulation ${ }^{7,8}$, and the ability to inhibit the expression of inflammatory factor protein levels and other functions ${ }^{9}$. PPARG is the coding gene of peroxisome proliferator-activated receptor gamma, and a ligand-dependent transcription factor. Distinguishing from the classical receptors which implant into the cell membrane and active cell signal cascade reaction to exercise function, PPARG inhibits in the expression of genes related to inflammation immediately ${ }^{10}$. A large number of studies have confirmed that the activation of PPARG is a significant target of osteoarthritis (OA) ${ }^{11}$. PPAR $\gamma$ agonists can inhibit inflammation and reduce cartilage degradation products in vivo and in vitro, and delay the progress of OA in model animals. Therefore, PPAR $\gamma$ is the key regulator of normal cartilage state; lack of it will lead to the spontaneous exacerbation of OA phnotype. MAPK1 (MitogenActivated Protein Kinase 1) is an encoding gene of protein MAPK1, Mitogen-activated protein kinase (MAPK or MAP kinase) is a protein kinase specific for the amino acids serine and 
threonine. Serine/threonine kinase which acts as an essential component of the MAP kinase signal transduction pathway. Depending on the cellular context, the MAPK/ERK cascade mediates diverse biological functions such as cell growth, adhesion, survival and differentiation through the regulation of transcription, translation, cytoskeletal rearrangements. MAPK1 is involved in directing cells to respond to various stimulation, such as mitogen, osmotic stress, heat shock protein and pro-inflammatory cytokines ${ }^{12}$.

In this project, we used network pharmacology to speculate that the active chemical compound piperine in piper longum $\mathrm{L}$ is its target for treating knee osteoarthritis (KOA), and then made attempts to find out its anti-KOA targets genes, using molecular biological methods for verification.

\section{MATERIALS AND METHODS}

\subsection{Collection and Screening of Candidate Active Compounds in Piper longum L}

Through the traditional Chinese medicine database TCMSP (http://sp.nwu.edu.cn/tcmsp.php) and TCM @ TAIWAN Taiwan Traditional Chinese Medicine Database (http://tcm.cmu.edu.tw/index.php) to search for active compounds related to piper longum L, and collect with the summary of the main chemical components retrieved from TCMID (http://www.Megabionet.Org/tcmid/) and TCM-PTD (http://tcm.zju.edu.cn/) and other databases, combining with the comprehensive literature retrieval and mining results from PALM-IST (www.hpppi.iicb.res.in), COREMINE (www.coremine.com), POLYSERCH and other websites, and confirms the molecule structure of each active compound through TCMSP 
and Pub Chem (https://pubchem.ncbi.nlm.nih.gov/) websites ${ }^{13,14}$. Utilizing the TCMSP database, we assessed the pharmacokinetics (Absorption, Distribution, Metabolism, Excretion, ADME) of major compound components according to Lipinski's rule, the chemical composition whose oral bioavailability (OB) $\geq 40 \%$ and drug-like (Drug-likeness, DL) $\geq 0.18$ were elected as candidate active ingredients.

\subsection{Prediction of Potential Targets}

The strength of the interaction between the active ingredient and protein is represented by the docking score (the Z-score). The DRAR-CPI server (https://cpi.biox.cn/drar/) adopts the bidirectional Z-transform function conversion to the Z-score in order to improve the accuracy of the prediction. The server recommends that the Z-score $<-0.5$ of the drug molecule-protein interaction should be included as potential targets. Therefore, the screened mol2 format files or SDF format files of the piper longum L candidate active molecule are uploaded to the server ${ }^{15}$. Download the computational analysis and set the protein targets inclusion standard to Z-score<1 to further reduce the range and enhance the prediction accuracy. Imported the PDBID of the screened protein targets to Unipot database (http://www.uniprot.org/), and the gene targets of the piper longum $\mathrm{L}$ were obtained by searching and transformation.

\subsection{Collection of Knee Osteoarthritis (KOA) Related Targets}

Anti-knee osteoarthritis (KOA) drug targets were collected from DisGeNET (http://www.disgenet.org/web/Dis-GeNET/menu), OMIM database (https://omim.org/), and CTD database (http://ctd. Mdibl.Org/), and then we established a target database related to knee osteoarthritis diseases combing with literature mining ${ }^{16}$. Utilizing comparative analysis 
between the above-mentioned piper longum L co-targets and knee osteoarthritis (KOA) related targets, we summarized the predicted targets with against KOA notably, and finally established the target genes library of piper longum $\mathrm{L}$ anti-KOA ${ }^{17}$.

\subsection{Protein and Genetic Information Correction}

Since the targets retrieved may have irregular names or contain proteins or genes from different species, all of the target information collected at each step mentioned above has been done with the normative approach. The specific method is as follows: Using the UniProtKB search function in the UniProt database (http://www.uniprot.org/), and importing the protein name and limit the species to humans (Homo sapiens), we gave all the retrieved proteins their official names (Official Symbol) and extracted the standard gene names ${ }^{18}$. We got the right target information by database retrieval and transformation ${ }^{19}$.

\subsection{Genetic Analysis and Pathway Annotation}

Our experiment used the Swiss target prediction function (http://www.swisstargetprediction.ch/), and we performed gene function analysis and KEGG pathway annotation on the co-targets of piper longum $\mathrm{L}$ anti-KOA by using a molecule annotation system ${ }^{20}$. We mapped the gene, which corresponded to the regulatory target protein to the pathway directly. and considered the pathway enriched by the drug target to be a pathway for drug regulation ${ }^{21}$. By the piper longum L-target-pathway network map (Composite-TargetPathway network map), co-targets of piper longum L anti-KOA were analyzed, the synergistic effect and superposition effect between drugs and pathways were identified ${ }^{22}$. 


\subsection{Macromolecular Docking}

Molecular docking is a computational model of the quaternary structure of a complex formed by two or more interacting biological macromolecules. It fully reveals the biological combination of protein and compound molecules. We used piperine as a ligand and PPARG protein as a receptor for molecular docking ${ }^{23}$. The docking was mainly done by AutoDock 4.2.6. We used the ZINC database (http://zinc.docking.org/) to download the 3D structure file of the active compound. The ligand and receptor molecules need to be energy-minimized before docking, then we deleted the receptor molecules (in the PDB file), added polar hydrogen atoms, electric charges, and magnetic field. We took the position of the original ligand compound as the binding site, and took all substructures with a radius of $0.65 \mathrm{~nm}$ as the active pocket part of the binding site. Autodock molecular docking software (version 2.5) was used to dock the active ingredients of Mongolian medicine with receptor protein molecules to screen the active ingredients of Piper longum L.

\subsection{Piperine Intervention for Knee Osteoarthritis Rat Models Caused by Knee Papain}

\section{Injection}

54 male SD rats (200 250 g, Beijing Vital River Laboratory Animal Technology Co., Ltd. , Beijing, China) were randomly divided into (1) Blank control group $(\mathrm{n}=18)$ (2) Knee osteoarthritis model group (Model group) $(\mathrm{n}=18)$ : Rats were fasted and deprived of water for 12 hours before the experiment. After weighing, rats were anesthetized by intraperitoneal injection with $1 \%$ sodium pentobarbital $(0.40 \sim 0.50 \mathrm{ml} / 100 \mathrm{~g})$, and were supine on a wooden board, and the prepared skin of $1 \mathrm{~cm}$ area around double knee joints was disinfected with 
complexed iodine. The knees of the rats were bent. Used the knee eye on the outer edge of the white patellar tendon at the lower end of the patella as the entry point, punctured the needle in the direction of the fossa intercondylar, and retracted a little after reaching the condyles of the femur. The $50 \mu \mathrm{L}$ micro-syringe was used to inject a mixture of papain (Sigma-Aldrich Co., Ltd, Beijing, China.) and L-cysteine (Sigma-Aldrich Co., Ltd, Beijing, China.) into the bilateral knee joints cavity at a dose of $0.1 \mathrm{~mL} / \mathrm{kg}$. (Preparing papain, L-cysteine powder and normal saline to a concentration of $4 \%$ and $0.03 \mathrm{~mol} / \mathrm{L}$ respectively under sterile conditions in advance, and storing them in a refrigerator at $4{ }^{\circ} \mathrm{C}$, taking them out $4 \mathrm{~h}$ in advance, and placing them to room temperature. Before the experiment, mix the $4 \%$ papain solution and $0.03 \mathrm{~mol} / \mathrm{L} \mathrm{L}$ cysteine solution prepared in advance at a ratio of 2: 1, and then perform knee joint cavity injection in half an hour.) The wounds were disinfected with iodophors after injection and routine care was performed. On the 4th and 7th days, two more intra-articular injections were performed in the same way. (3) The Piperine Group ( $\mathrm{n}=18)$ It is converted into the dose of rats according to body weight, and the dose of intragastric administration of rats was calculated as $10 \mathrm{ml} / \mathrm{kg}$. On the basis of the knee osteoarthritis model group, the piperine group began to receive a corresponding dose of piperine (Western Chemical Science and Technology Co., Ltd., Beijing, China) intragastrical instillation every week starting from the 2nd week, and the experiment was completed at 20 weeks. The other two groups were given the same dose of saline.

\subsection{Western Blot Analysis of PPARG and MAPK1 Expression Levels}

Collected cartilage tissue around the knee joints of each group of rats, lysed histiocytes, extracted cytoplasm and nuclear protein supernatant, and ran SDS-PAGE gel. Applied constant 
pressure $120 \mathrm{~V}$, $2 \mathrm{~h}$ electrophoresis before the wet transfer method. Electrotransfered to PVDF membrane, in a blocking solution of 5\% skim milk TBST, at room temperature for 1 hour, and added primary antibody (Cell Signaling Technology, USA) after TBST rinse. Shaked the mixture overnight at $4^{\circ} \mathrm{C}$. The TBST solution was rinsed several times. Added rabbit antihuman related protein polyclonal antibody, shaken at room temperature for 1 hour, rinsed three times, developed ECL color and detected PPARG and MAPK1 expression.

\subsection{Immunohistochemistry and $\mathrm{HE}$}

$4 \mu \mathrm{m}$ continuous section of the representative wax blocks of cartilage tissue around the knee joint was taken for routine dewaxing and hydration. Incubate inactivated endogenous peroxidase with $3 \%$ hydrogen peroxide in methanol solution, microwave heat-repaired antigen, and rabbit anti-human PPARG and MAPK1 gene polyclonal antibodies were incubated at $4{ }^{\circ} \mathrm{C}$ overnight. Phosphate buffer was used to substitute the primary antibody as a negative control. En Vjsion Detec60n kit was used for immunohistochemical staining, and dimethylbenzidine (DAB) was used for color development. Conventional hematoxylin counterstained, dehydrated, transparent, and mounted. The secondary antibody and immune tissue immunofluorescence double standard chemical staining were performed according to the UltraSensitiveTM SP kit instructions. The cartilage tissue was paraffin embedded and HE stained.

\subsection{Immunoprecipitation Experiment}

SD rat knee joint chondrocytes were cultured in RPMI1640 medium containing 10\% BCS and passaged into $10 \mathrm{~cm}$ petri dishes. Using Supeffect transfection reagent, PPARG plasmid (pcDNA3.0-flag-PPARG) and MAPK1 plasmid (pCMV-Myc-MAPK1) (Ruibo Biotechnology, 
Guangzhou, China) were co-transfected into chondrocytes from SD rat knee joints, cells were harvested $48 \mathrm{~h}$ after transfection and total cell proteins were extracted. Mixed the protein supernatant with $2 \mu \mathrm{g}$ of antibody anti-myc, and incubated for $3 \mathrm{~h}$ at $4{ }^{\circ} \mathrm{C}$ with gently shaking, added $40 \mu \mathrm{L}$ Protein $\mathrm{A} / \mathrm{G}$ agarose beads, and incubated for $8 \mathrm{~h}$ at $4{ }^{\circ} \mathrm{C}$ with gently shaking, and then coupled antibodies with agarose. Centrifuged at $4{ }^{\circ} \mathrm{C}$ and eluted the beads 3 times with lysate. Added $2 \times \operatorname{SDS}$ loading buffer for 5 minutes at $100^{\circ} \mathrm{C}$, loaded on $10 \%$ SDS-PAGE gel, transferred the membrane by electrophoresis. Western Blot experiments using Flag antibody were performed to detect the presence of Flag-PPARG in the complex. The MAPK1 plasmid (pCMV-Myc-MAPK1) transfected separately and the PPARG plasmid (pcDNA3.0-flagPPARG) transfected separately were set as experimental control groups.

\subsection{Statistical Methods}

The experimental data was expressed as mean \pm standard deviation (mean $\pm \mathrm{SD}$ ); the difference between the two groups was examined by Student's t test. Comparison of enumeration data between groups was performed using the chi-square test or Fisher's exact probability method. $\mathrm{P}<0.05$ was considered the statistical difference, and $\mathrm{P}<0.01$ was considered the difference to be statistically significant. Statistical analysis was performed using SPSS 18.0 software.

\section{RESULTS}

\subsection{Collection and Screening of Candidate Active Compounds in Piper Longum L}

Based on the traditional Chinese medicine database TCMSP, which contains piper longum Lrelated active compounds, the main chemical components in piper longum L were collected combinating with databases such as TCMID and TCM-PTD, and the molecular structure of 
each active compound was confirmed. The pharmacokinetics (Absorption, Distribution, Metabolism, Excretion, ADME) properties of the main compound components were evaluated, and 9 candidate compounds were obtained, which were selected to meet Lipinski's rule (The molecular weight was less than 500; the number of hydrogen bond donors was less than 5; the number of hydrogen bond acceptors was less than 10; the lipid-water partition coefficient was less than 5; the number of rotatable bonds was no more than 10). 7 compounds were obtained (TableIgreen). Piperine, which has moderate indexes, was selected as the candidate drug for this trial (Fig.1)

TableI. Collection and screening of candidate active compounds in piper longum $L$

\begin{tabular}{|c|c|c|c|c|c|c|c|c|}
\hline & $\begin{array}{l}\text { Chem } \\
\text { ical } \\
\text { Formula }\end{array}$ & $\begin{array}{r}\mathrm{M} \\
\text { olecula } \\
\mathrm{r} \\
\text { Weight }\end{array}$ & $\begin{array}{r}\mathrm{M} \\
\text { olecula } \\
\mathrm{r} \\
\text { Volum } \\
\mathrm{e}\end{array}$ & $\begin{array}{c}\text { Log } \\
\mathrm{P}\end{array}$ & $\begin{array}{l}\text { Molec } \\
\text { ular Polar } \\
\text { Surface } \\
\text { Area }\end{array}$ & $\begin{array}{c}\mathrm{N} \\
\text { umber } \\
\text { of } \\
\text { Hydro } \\
\text { gen } \\
\text { Bond } \\
\text { Accept } \\
\text { ors }\end{array}$ & $\begin{array}{l}\mathrm{N} \\
\text { umber } \\
\text { of } \\
\text { Hydro } \\
\text { gen } \\
\text { Bond } \\
\text { Donor } \\
\mathrm{s}\end{array}$ & $\begin{array}{r}\mathrm{Nu} \\
\text { mber of } \\
\text { Rotatable } \\
\text { Bonds }\end{array}$ \\
\hline $\begin{array}{l}\qquad 1,2,15,16- \\
\text { Tetrahydrotanshiquinone }\end{array}$ & $\mathrm{C}_{18} \mathrm{H}_{16} \mathrm{O}_{3}$ & $\begin{array}{l}280.31 \\
8\end{array}$ & 181.44 & .305 & 43.37 & 3 & 0 & 0 \\
\hline Isobutylisovalerate & $\mathrm{C}_{9} \mathrm{H}_{18} \mathrm{O}_{2}$ & $\begin{array}{l}158.23 \\
8\end{array}$ & 127.59 & .587 & 26.3 & 2 & 0 & 5 \\
\hline Lawsone & $\mathrm{C}_{10} \mathrm{H}_{6} \mathrm{O}_{3}$ & $\begin{array}{l}174.15 \\
3\end{array}$ & 100.49 & .22 & 54.37 & 3 & 1 & 0 \\
\hline Piperine & $\mathrm{C}_{17} \mathrm{H}_{19} \mathrm{NO}_{3}$ & $\begin{array}{l}285.33 \\
8\end{array}$ & 186.93 & .864 & 38.77 & 3 & 0 & 3 \\
\hline Piperitenone & $\mathrm{C}_{10} \mathrm{H}_{14} \mathrm{O}$ & $\begin{array}{l}150.21 \\
8\end{array}$ & 115.59 & .063 & 17.07 & 1 & 0 & 0 \\
\hline
\end{tabular}




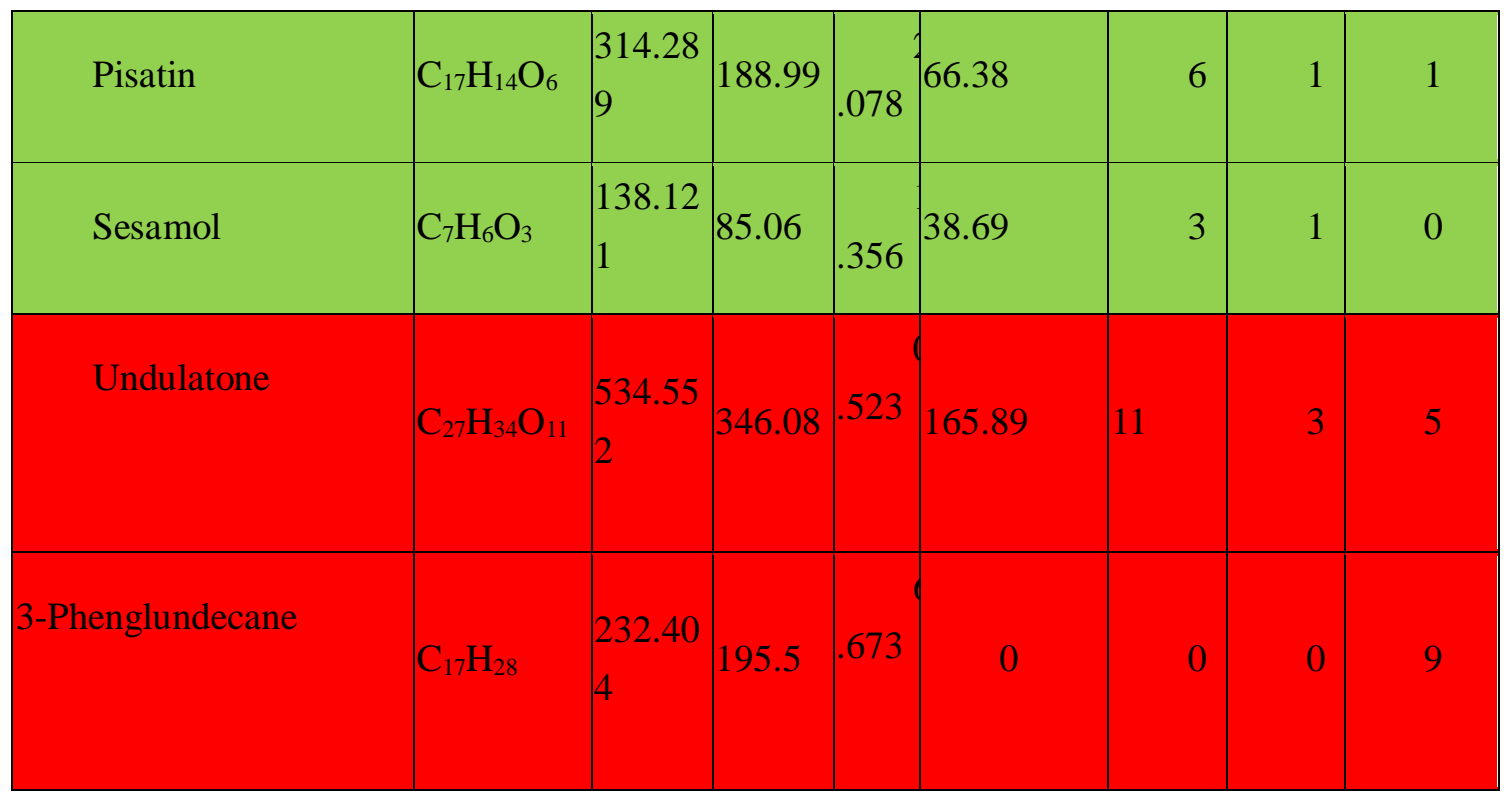

\subsection{Piperine Cell Signaling Pathway Annotation}

The Swiss target prediction function (http://www.swisstargetprediction.ch/) was used in this experiment, and the biomolecular function annotation system was used to perform gene function analysis on the co-targets between piperine and piper longum L against knee osteoarthritis. Using DisGeNET (http://www.disgenet.org/web/Dis-GeNET/menu), OMIM (https://omim.org/), and CTD (Http: // ctd. Mdibl.Org/) databases to collect targets treating knee osteoarthritis, 20 highly overlapping signal pathways were found. The osteoarthritis-related KEGG pathway annotations were selected, and the genes corresponding to piperine's regulatory target proteins were directly mapped to the pathways, and the above-mentioned collected piper longum L co-targets were compared with the KOA-related targets(Fig. 1).

Fig. (1). A: Piperine chemical structure; B: The heat map of pathway or biological process enrichment; C: Pathways or biological processes classified by functional relevance; D:

Degree of the pathway or biological process enrichment; Piperine's cell signaling pathway 
annotation: Piperine's interventional disease database GO function database and KOA GO function database were collected from the DisGeNET database, OMIM database and CTD database, respectively.

\subsection{Prediction of Potential Anti-OA Targets of Piperine}

Selected drug-protein interactions as potential targets (Fig. 2A). The PDBID of the selected protein targets was imported into the UniProt database (https://www.Uniprot.Org/), and the gene targets of the piper longum L components were obtained through searching and transformation (Fig. 2B). Collected the drug targets for knee osteoarthritis from DisGeNET (http://www.disgenet.org/web/Dis-GeNET/menu) and CTD (https://ctd. Mdibl.Org/) databases

(Fig. 2C). Normalized target information. Obtained correct target information through database retrieval and transformation. There were three target proteins PPARG, RXRA, and MAPK1 that are related to each other (Fig. 2D).

Fig. (2). Prediction of Potential Anti-OA Targets of Piperine. A: Selecting drug moleculeprotein interactions as potential targets; B: Importing the PDBID of the screened protein target into the UniProt database to obtain the gene targets of the Piper longum L components; C: Collection of drug targets for the treatment of knee osteoarthritis from DisGeNET and CTD databases; D: Standardization of the target information to obtain the correct target information. There are three target proteins that are correlated.

\subsection{The Results of Molecular Docking}


We validated the key active compound piperine screened by network pharmacology, then imported its 3D structure into AutoDock and connected with PPARG. Their interaction with the surrounding key amino acids and their binding at the active site are shown in Figure 3. The results show that piperine and PPARG's ARG-212 and GLN-420 amino-acid residues are mainly combined in the form of hydrogen bonds which have good binding activity. It shows that piperine can hinder the combination of the ARG-212 and GLN-420 domains of PPARG protein, and the docking energy value is smaller and the binding is more stable. The above results indicate that using piperine in combination with PPARG may effectively increase the expression of PPARG and thus inhibit the inflammatory process.

Fig. (3). The results of simulating the combination of piperine and PPARG in vivo from all angles using AutoDock software

\subsection{Pathology Verification}

The HE staining showed that the cartilage tissue of the model control group appeared inflammatory changes, which showed obvious expansion of capillaries in the perichondrium, thickening of the vascular wall, irregular arrangement of chondrocytes and atrophy of chondrocytes. Inflammatory cell infiltration was observed. Compared with the model group, the cartilage tissue of the rats in the piperine group showed no obvious inflammatory changes, the capillaries in the cartilage were not significantly dilated, the vascular wall was thin, the inflammatory cell infiltration was not obvious, and the chondrocytes showed a single distribution, small size, flat; the closer to the center of cartilage, the more mature the chondrocytes, the larger the volume; round or oval, round nucleus, visible nucleoli, weak basophilic cytoplasm. Homologous cell population can be seen, the place close to the cartilage 
pit was strongly basophilic, and the cartilage sac surrounded the chondrocytes (Fig. 4A). Immunohistochemical results showed that PPARG protein was expressed at a low level in the cartilage tissue of the KOA model group, and the positive expression rate of PPARG protein in the piperine treatment group was significantly increased $(\mathrm{P}<0.05)$. Compared with the model group, the positive expression rate of MAPK1 protein in cartilage tissue of the piperine treatment group was significantly decreased. PPARG protein was expressed in the nucleus and cytoplasm, mainly in the cytoplasm, MAPK1 was expressed in the cytoplasm, and the two had the possibility of co-expression (Fig. 4B).

Fig. (4). Pathological effect of piperine of knee osteoarthritis model rats. A:HE staining showed that the KOA model group had significantly dilated capillaries, irregular arrangement of chondrocytes, and more inflammatory cells infiltration. In the piperine group, there was no apparent dilation of capillaries, a certain regularity of chondrocytes in the cartilage, and no more inflammatory cell infiltration; B:Immunohistochemical results showed that compared with the model group, the positive expression rate of PPARG protein in cartilage tissue of Piperine treatment group was increased to varying degrees; compared with the model group, the positive expression rate of MAPK1 in cartilage tissue of Piperine treatment group was decreased to varying degrees.

\subsection{Western Blot and Co-immunoprecipitation Demonstrated the Association of PPARG} and MAPK1

Western-blot results showed that at 20th weeks, the expression of MAPK1 in Piperine group 
was significantly lower than it at 4 th weeks $(\mathrm{P}<0.01)$, and the expression of PPARG increased with the Piperine administration time $(\mathrm{P}<0.01)$ (Fig. 5A). Co-immunoprecipitation experiment co-transfected pCMV-Myc-MAPK1 plasmid and pcDNA3.0-flag-PPARG plasmid into SD rats knee chondrocytes. Co-immunoprecipitation was performed with the antibody Myc, and Western blot experiments were performed with the antibody Flag. The results showed that the presence of PPARG was detected in the experimental group, and no PPARG was detected in the negative control group. (Fig. 5B). The above experiment was repeated three times under the same conditions, and the same results were obtained. Experiments showed that PPARG protein and MAPK1 protein can specifically bind, and the two proteins interact in the cell.

Fig. (5). Western blot and co-immunoprecipitation. A: Western-blot results showed that at 20th weeks, the expression of MAPK1 in piperine group was significantly lower than it at 4th weeks ( $\mathrm{P}<0.01$ ), and the expression of PPARG increased significantly with the administration time of piperine $(\mathrm{P}<0.01)$; B. Co-immunoprecipitation showed that the presence of PPARG was detected in the experimental group and no PPARG was detected in the negative control group, indicating that the PPARG protein can specifically bind to the MAPK1 protein.

\section{DISCUSSION}

Knee osteoarthritis can be caused by factors such as humidity, cold wind, working in cold water for a long time, strenuous activity, hyperhypnosis, eating greasy food, less movement, and overwork $^{24}$. Osteoarthritis pain is a kind of non-protective pain ${ }^{25}$. It can induce pain when the affected joints are passively moved. It mostly affect the elderly. If it is not treated in time, it 
will cause joint deformity and disability ${ }^{26}$. Mongolia medicine Naru Sanwei Pill is composed of Radix Aconiti Kusnezoffii Praeparata, Medicine Terminalia Fruit and Piper Longum L. It has the effects of convergence and detoxification, promoting blood circulation and removing blood stasis, immunoregulation, relieving swelling and pain. It is used to treat diseases such as knee osteoarthritis increasingly more and more. Earlier studies found that Mongolian medicine Naru Sanwei Pill has anti-inflammatory effects on osteoarthritis as a natural medicine ${ }^{27}$. In the prescription, the anti-inflammatory and analgesic medicine piper longum $\mathrm{L}$ is the main medicine ${ }^{28}$. Although studies have confirmed that aconitine, piperine, and gallic acid are the main blood entering component of Mongolian medicine Naru Sanwei Pills, the question of which ingredient is the main compound for the treatment of knee osteoarthritis in piper longum L has not been solved. This project used network pharmacological methods to predict and analyze the target of effective components in the treatment of knee osteoarthritis and used protein imprinting and co-immunoprecipitation to verify piperine's targets anti-knee osteoarthritis.

PPARG is a gene encoding peroxisome proliferator-activated receptor $\gamma$ and a ligand-activated transcription factor. The protein encoded by PPARG gene belongs to the nuclear receptor peroxisome proliferator-activated receptor subfamily. Recent studies have found that PPAR- $\gamma$ can regulate inflammation and participate in the occurrence and development of many chronic immunopathy. PPARG can reduce the inflammatory response by inhibiting the NF- $\kappa \mathrm{B}$ signaling pathway. PPAR $\gamma$ has three main functions: adipocyte differentiation, insulin sensitivity, and lineage assignment of mesenchymal stem cells (MSC), all three of which are ligand-dependent. Chondrocytes, fibroblasts, osteoblasts, and adipocytes originate from the same bone marrow 
mesenchymal stem cells (MSC). PPARG plays a guiding role in the differentiation and lineage allocation of MSCs ${ }^{29}$. All subtypes of the PPAR family have been identified in joints or inflammatory cells ${ }^{30}$. Members of the PPAR family function by joining together with retinoid $\mathrm{X}$ receptors (RXR) to form heterodimers, which can combine with specific DNA sequences to regulate the transcription of multiple genes, and this specific DNA sequence is often called peroxisome proliferative response element (PPRE). PPARG has the ability to directly inhibit the expression of inflammatory cytokines and the differentiation of immune cells to the antiinflammatory phenotype, and plays a fundamental role in the immune response ${ }^{31}$. Its activation leads to transcription inhibition of pro-inflammatory cytokines (IL-1, TNF $\alpha$ ), early inflammatory genes (NOS (2), COX-2, mPGES-1) or transcriptional repression of matrix metalloproteinases (MMP-1, MMP-13). PPAR $\gamma$ agonists inhibit inflammation and reduce the synthesis of cartilage degradation products in vitro and in vivo, significantly reduce the expression of crucial catabolism markers and inflammation markers, can reduce synovial inflammation, and prevent cartilage destruction or inflammatory cartilage dissolution ${ }^{32}$. PPAR $\gamma$ deficiency up-regulates mTOR signaling, resulting in reduced autophagy inhibition and chondroprotective effects reduction and increased catabolic activity. MAPK1 (mitogenactivated protein kinase 1) is a protein-coding gene. The gene encodes a MAP kinase, also called extracellular signal-regulated kinase (ERK), which acts as an integration point for multiple biochemical signals and participates in a variety of cellular processes, such as proliferation, differentiation, transcriptional regulation, and development ${ }^{33}$. MAPK1 / ERK2 and MAPK3 / ERK1 are two MAPKs that play an essential role in the MAPK / ERK cascade ${ }^{34}$. They also participate in signal cascades initiated by activated KIT and KITLG / SCF. 
Depending on the cell context, the MAPK / ERK cascade mediates multiple biological functions, such as cell growth, adhesion, survival, and differentiation, by regulating transcription, translation, and cytoskeletal rearrangement. The MAPK / ERK cascade also plays a role in the initiation and regulation of meiosis, mitosis, and post-mitotic function by phosphorylating many transcription factors ${ }^{35}$.

In the knee osteoarthritis rat models treated by Mongolian piperine, the expression of PPARG was up-regulated, but the expression of MAPK1 was down-regulated. Therefore, we speculated that PPARG might reversely regulate the MAPK1 gene, and Co-immunoprecipitation confirmed direct regulation between the two. Based on the above bioinformatics screening and preliminary experiments, the direct regulatory relationship between PPARG and MAPK1 may be the biological mechanism of knee osteoarthritis development.

This experiment proves that Piper longum L/ Piperine / PPARG / MAPK1 plays an essential role in regulating the occurrence and development of knee osteoarthritis, and provides new targets for the treatment of knee osteoarthritis in the future. It reflects the characteristics of Mongolian medicines with enormous ingredients, numerous targets, and voluminous pathways synergistic effects, which helps to solve the problems of knee osteoarthritis and a series of neuropathic pain. It provides the experimental basis for later research and development of efficient, specific and targeted drugs for the treatment of knee osteoarthritis and provides useful experience for the development of national drugs. The specific mechanism of piperine to the two proteins is not precise, and needs further researching.

\section{CONCLUSION}


This experiment proves the important regulatory role of piper longum L / piperine/ PPARG / MAPK1 in the occurrence and development of knee osteoarthritis. It provides new targets for the treatment of knee osteoarthritis in the future and helps to provide new methods and ideas for the treatment of inflammation. It provides experimental basis for the research and development of highly effective, specific and targeted drugs for knee osteoarthritis, and provides useful experience for the development of national medicines.

\section{ACKNOWLEDGEMENTS}

This research is funded by the following projects: National Natural Science Foundation of C hina (No. 81760748 and 81550047); the Major Science Foundation of Affiliated Hospital of I nner Mongolia Medical University(NYFY ZD 010); Natural Science Foundation of Inner Mo ngolia Autonomous Region(No.2019MS08110,2018LH08032 and 2017MS0831); Inner Mo ngolia Autonomous Region "Prairie excellence"Project.Western Light Young Scholars Prog ram of the Chinese Academy of Sciences.

\section{CONFLICT OF INTEREST}

We declare that we have no financial and personal relationships with other people or organizations that can inappropriately influence our work, there is no professional or other personal interest of any nature or kind in any product, service and/or company that could be construed as influencing the position presented in, or the review of, the manuscript entitled. 


\section{ETHICS STATEMENT}

The animal experiments in this study followed the guidelines of the Ethics Committee of the Affiliated Hospital of Inner Mongolia Medical University on the care and use of laboratory animals.

\section{REFERENCE}

1. Martel-Pelletier J, Barr AJ, Cicuttini FM, et al. Osteoarthritis. Nat Rev Dis Primers. 2016;2:16072.

2. Conaghan PG, Cook AD, Hamilton JA, Tak PP. Therapeutic options for targeting inflammatory osteoarthritis pain. Nat Rev Rheumatol. 2019;15(6):355-363.

3. Jones IA, Togashi R, Wilson ML, Heckmann N, Vangsness CT. Intra-articular treatment options for knee osteoarthritis. Nat Rev Rheumatol. 2019;15(2):77-90.

4. Chevalier X, Eymard F, Richette P. Biologic agents in osteoarthritis: hopes and disappointments. Nat Rev Rheumatol. 2013;9(7):400-410.

5. He H, Guo WW, Xu RR, et al. Alkaloids from piper longum protect dopaminergic neurons against inflammation-mediated damage induced by intranigral injection of lipopolysaccharide. BMC Complement Altern Med. 2016;16(1):412.

6. Yadav V, Krishnan A, Vohora D. A systematic review on Piper longum L.: Bridging traditional knowledge and pharmacological evidence for future translational research. J Ethnopharmacol. 2020;247:112255.

7. Ren C, Liang Z. Piperine alleviates lipopolysaccharide-induced inflammatory injury by 
down-regulating microRNA-127 in murine chondrogenic ATDC5 cells. Biomed Pharmacother. 2018;103:947-954.

8. Ying X, Chen X, Cheng S, Shen Y, Peng L, Xu HZ. Piperine inhibits IL- $\beta$ induced expression of inflammatory mediators in human osteoarthritis chondrocyte. Int Immunopharmacol. 2013;17(2):293-299.

9. Hu D, Wang $Y$, Chen $Z$, et al. The protective effect of piperine on dextran sulfate sodium induced inflammatory bowel disease and its relation with pregnane $\mathrm{X}$ receptor activation. J Ethnopharmacol. 2015;169:109-123.

10. Chawla A, Barak Y, Nagy L, Liao D, Tontonoz P, Evans RM. PPAR-gamma dependent and independent effects on macrophage-gene expression in lipid metabolism and inflammation. Nat Med. 2001;7(1):48-52.

11. Kawai M, Sousa KM, MacDougald OA, Rosen CJ. The many facets of PPARgamma: novel insights for the skeleton. Am J Physiol Endocrinol Metab. 2010;299(1):E3-9.

12. Murphy LO, Blenis J. MAPK signal specificity: the right place at the right time. Trends Biochem Sci. 2006;31(5):268-275.

13. Kim S, Thiessen PA, Bolton EE, et al. PubChem Substance and Compound databases. Nucleic Acids Res. 2016;44(D1):D1202-1213.

14. Ru J, Li P, Wang J, et al. TCMSP: a database of systems pharmacology for drug discovery from herbal medicines. J Cheminform. 2014;6:13.

15. Luo H, Chen J, Shi L, et al. DRAR-CPI: a server for identifying drug repositioning 
potential and adverse drug reactions via the chemical-protein interactome. Nucleic Acids Res. 2011;39(Web Server issue):W492-498.

16. Amberger JS, Bocchini CA, Schiettecatte F, Scott AF, Hamosh A. OMIM.org: Online Mendelian Inheritance in Man (OMIM®), an online catalog of human genes and genetic disorders. Nucleic Acids Res. 2015;43(Database issue):D789-798.

17. Piñero J, Ramírez-Anguita JM, Saüch-Pitarch J, et al. The DisGeNET knowledge platform for disease genomics: 2019 update. Nucleic Acids Res. 2019.

18. Boutet E, Lieberherr D, Tognolli M, et al. UniProtKB/Swiss-Prot, the Manually Annotated Section of the UniProt KnowledgeBase: How to Use the Entry View. Methods Mol Biol. 2016;1374:23-54.

19. Poux S, Arighi CN, Magrane M, et al. On expert curation and scalability: UniProtKB/Swiss-Prot as a case study. Bioinformatics. 2017;33(21):3454-3460.

20. Daina A, Michielin O, Zoete V. SwissTargetPrediction: updated data and new features for efficient prediction of protein targets of small molecules. Nucleic Acids Res. 2019;47(W1):W357-w364.

21. Wu Y, Zhang F, Yang K, et al. SymMap: an integrative database of traditional Chinese medicine enhanced by symptom mapping. Nucleic Acids Res. 2019;47(D1):D1110-d1117.

22. Zoete V, Daina A, Bovigny C, Michielin O. SwissSimilarity: A Web Tool for Low to Ultra High Throughput Ligand-Based Virtual Screening. J Chem Inf Model. 
2016;56(8):1399-1404.

23. Forli S, Huey R, Pique ME, Sanner MF, Goodsell DS, Olson AJ. Computational protein-ligand docking and virtual drug screening with the AutoDock suite. Nat Protoc. 2016;11(5):905-919.

24. Berenbaum F, Wallace IJ, Lieberman DE, Felson DT. Modern-day environmental factors in the pathogenesis of osteoarthritis. Nat Rev Rheumatol. 2018;14(11):674681.

25. Hunter DJ, Bierma-Zeinstra S. Osteoarthritis. Lancet. 2019;393(10182):1745-1759.

26. Felson DT. Osteoarthritis: priorities for osteoarthritis research: much to be done. Nat Rev Rheumatol. 2014;10(8):447-448.

27. Nirmal P, Koppikar S, Bhondave P, et al. Influence of six medicinal herbs on collagenase-induced osteoarthritis in rats. Am J Chin Med. 2013;41(6):1407-1425.

28. Xiao Y, Shi M, Qiu Q, et al. Piperlongumine Suppresses Dendritic Cell Maturation by Reducing Production of Reactive Oxygen Species and Has Therapeutic Potential for Rheumatoid Arthritis. J Immunol. 2016;196(12):4925-4934.

29. Wahli W, Michalik L. PPARs at the crossroads of lipid signaling and inflammation. Trends Endocrinol Metab. 2012;23(7):351-363.

30. Moraes LA, Piqueras L, Bishop-Bailey D. Peroxisome proliferator-activated receptors and inflammation. Pharmacol Ther. 2006;110(3):371-385.

31. Lee CH, Evans RM. Peroxisome proliferator-activated receptor-gamma in 
macrophage lipid homeostasis. Trends Endocrinol Metab. 2002;13(8):331-335.

32. Wang $\mathrm{Y}$, Zhao X, Lotz M, Terkeltaub R, Liu-Bryan R. Mitochondrial biogenesis is impaired in osteoarthritis chondrocytes but reversible via peroxisome proliferatoractivated receptor y coactivator 1a. 2015;67(8):2141-2153.

33. Jeon $\mathrm{OH}$, Kim C, Laberge RM, et al. Local clearance of senescent cells attenuates the development of post-traumatic osteoarthritis and creates a pro-regenerative environment. Nat Med. 2017;23(6):775-781.

34. Cobb MH, Hepler JE, Cheng M, Robbins D. The mitogen-activated protein kinases, ERK1 and ERK2. Semin Cancer Biol. 1994;5(4):261-268.

35. Ohnishi H, Takeda K, Domenico J, et al. Mitogen-activated protein kinase/extracellular signal-regulated kinase 1/2-dependent pathways are essential for CD8+ T cell-mediated airway hyperresponsiveness and inflammation. J Allergy Clin Immunol. 2009;123(1):249-257. 